\title{
Philosophiques
}

\section{Constructivisme et structuralisme dans les fondements des mathématiques}

\section{Yvon Gauthier}

Volume 1, numéro 1, avril 1974

URI : https://id.erudit.org/iderudit/203004ar

DOI : https://doi.org/10.7202/203004ar

Aller au sommaire du numéro

\section{Éditeur(s)}

Société de philosophie du Québec

ISSN

0316-2923 (imprimé)

1492-1391 (numérique)

Découvrir la revue

Citer cet article

Gauthier, Y. (1974). Constructivisme et structuralisme dans les fondements des mathématiques. Philosophiques, 1(1), 83-105. https://doi.org/10.7202/203004ar
Résumé de l'article

L'auteur a voulu définir deux orientations principales dans les recherches sur les fondements des mathématiques, le constructivisme et le structuralisme (entendu au sens bourbakiste de " théorie de structures abstraites") . Il montre à l'aide d'exemples tirés de la théorie axiomatique des ensembles, e.g. l'hypothèse du continu, et de l'intuitionnisme, e.g. la notion de séquence de choix, que les deux approches constituent des voies complémentaires dans les recherches sur les fondements. L'auteur propose quelques idées nouvelles, en particulier sur le continu et l'horizon constructif, tout au long de l'article et dans un appendice. L'article se résume à la défense et l'illustration d'une philosophie constructivisme en voie d'élaboration. 


\title{
CONSTRUCTIVISME ET STRUCTURALISME DANS LES FONDEMENTS DES MATHEMATIQUES *
}

\author{
par Yvon Gauthier
}

Mon exposé porte sur les fondements des mathématiques: je veux, dans une première partie, définir ce que j'entends par fondements des mathématiques; dans une deuxième partie, j'analyserai quelques problèmes fondationnels précis dans les perspectives structuraliste et constructiviste que j'aurai préalablement dégagées de la problématique des fondements. Ma conclusion indiquera quelques voies pour les recherches futures en fondements des mathématiques.

1. Je pense qu'il est peut-être utile tout d'abord de clarifier le statut de la problématique des fondements: l'expression fondements des mathématiques est employée souvent dans des sens différents, si ce n'est divergents. J'élimine tout de suite le sens «d'éléments fondamentaux », de mise en ordre ou d'organisation systématique d'un langage donné, d'un matériau mathématique précis; par exemple, l'ouvrage de Dieudonné Foundations of modern analysis ou celui d'Eilenberg et Steenrod Foundations of algebraic topology, ne traitent pas des fondements au sens que je définis à l'instant; les fondements des mathématiques constituent une analyse critique de la pratique mathématique et de sa validité, c'est-à-dire que les fondements prennent pour objet la pratique mathématique, les principes, les concepts fondamentaux des mathématiques. Les fondements des mathématiques sont une entreprise à la fois mathématique, logique et philosophique; c'est une discipline de synthèse qui utilise ces

* Le structuralisme dont je parle ici n'est pas celui des philosophes. Voir mon article «La notion théorique de structure», Dialectica 23 (1969). 
divers aspects logiques, mathématiques, et philosophiques ou épistémologiques dans un but théorique précis : la formulation d'une théorie de la pratique mathématique ${ }^{1}$. Notons que les fondements des mathématiques ne sont pas la philosophie des mathématiques, discipline plus ou moins anachronique dans son acception habituelle qui cherche à proposer du dehors une théorie ou des théories concernant la nature des êtres mathématiques et qui tente de décrire, avec plus ou moins de bonheur, la portée et les limites de la pensée mathématique. Notons aussi que les fondements ne coïncident pas avec la logique mathématique, avec laquelle on les confond souvent dans la littérature courante: si les résultats logico-mathématiques peuvent avoir une signification importante pour les fondements, ils sont souvent la suite de la pratique mathématique, plutôt que son analyse critique. Je dirai plus loin comment distinguer plus finement entre les fondements et la logique mathématique.

Cette définition préliminaire des fondements des mathématiques doit beaucoup à Georg Kreisel. C'est lui, le premier, qui a doté les fondements des mathématiques d'une spécificité assez grande pour en faire une discipline à part.

Historiquement les fondements ont été réduits ou bien à une certaine conception philosophique des mathématiques ou bien à un certain programme de reconstruction des mathématiques, à partir de la logique, par exemple, chez Frege et Russell. Sans doute, celui qui avait à l'origine la conception la plus précise et la plus radicale est Hilbert. On sait qu'il s'agissait pour lui d'assurer les mathématiques; il parle d'une «Sicherung», d'une certification sur des bases combinatoires finies d'objets concrets. On sait aussi que ce programme métamathématique a été contredit par les résultats d'incomplétude de Gödel. ${ }^{2}$ L'intuitionnisme de

1. Notons ici que nous donnons un sens précis - Kreisélien - à l'expression «fondements des mathématiques». Pour une perspective différente, yoir l'article de Leon HENKIN, «Mathematical Foundations for Mathematics》 dans American Matbematical Montbly, vol. 78, no. 5, pp. 463-487.

Ii est clair que nous rejetons l'appellation «fondements 》 pour l'ensemble des recherches logiques ou mathématiques qui portent sur des théories mathématiques fondamentales, mais qui n'ont pas de fonction fondationnelle comme telle.

2. Voir là-dessus G. KREISEL, «Hilbert's Programme», Dialectica, 12 (1958). 
Brouwer en admettant les objets abstraits, comme les fonctions et les fonctionnelles - ici je fais allusion à l'interprétation de Gödel «Ueber eine bisher noch nicht benützte Erweiterung des finiten Standpunktes» qui admet des fonctionnelles de type fini avec les seuls procédés de construction, de substitution et de récursion - va plus loin. Le formalisme élargi (où l'on accepte des méthodes constructives qui ne sont pas finitaires) et l'intuitionnisme ne sont pas incompatibles à la limite; ce sont des théories fondationnelles convergentes dans la mesure où la théorie des démonstrations débouche sur une théorie des constructions mentales, ce qu'est essentiellement l'intuitionnisme.

2. Mais avant de nous engager plus loin dans la discussion, il faut préciser le rôle de l'entreprise fondationnelle. Les fondements, pour être une analyse critique des principes et des concepts de la pratique mathématique, cherchent à asseoir l'ensemble des mathématiques sur des bases irréductibles, un langage primitif. L'idéal hilbertien de la certification ou de la solidification réapparaît ici, mais il est couplé à un système conceptuel où les notions primitives sont mises à jour. Cette volonté réductrice, et non pas « réductiviste», des fondements ne signifie pas qu'il faille réduire les mathématiques à leur plus simple expression, mais plutôt qu'il faut mettre à l'épreuve les notions fondamentales, par exemple, en tâchant d'étendre les mathématiques existantes dans des directions où les concepts et les principes de construction mathématique sont mis en relief comme, par exemple, dans la théorie des catégories et dans la théorie des topoi où ressort nettement le caractère géométrique ou algébraico-géométrique des constructions ${ }^{3}$.

J'appelle structurels les principes de construction fondés sur l'usage actuel et les extensions possibles d'un langage mathématique donné sans critiquer ce même langage et ses méthodes; sont donc constructifs ou constructivistes les principes de construc-

3. Voir G. KREISEL, «Perspectives in the Philosophy of Pure Mathematics》, à paraître. Voir aussi G. Kreisel «Observations on popular discussions of foundations》 in Axiomatic Set Tbeory, American Mathematical Society, (Rhode Island, 1971), pp. 189-197, où Kreisel s'en prend particulièrement à P.J. Cohen et A. Robinson. 
tion qui sont fondés sur une analyse critique du. langage utilisé; l'exemple le plus clair ici, la reconstruction intuitionniste de l'analyse classique. Je reviendrai là-dessus plus loin. Extension, validation, justification ou certification des principes, des méthodes et des concepts mathématiques constituent, par conséquent, le but même des fondements. Les fondements des mathématiques, pour préciser la définition que j'ai déjà proposée, sont une théorie générale des principes et des concepts mathématiques que l'analyse critique dégage de la pratique mathématique. Une telle théorie, il va sans dire, n'en est qu'aux premières ébauches; il faut se garder d'une première méprise qui consisterait à penser que l'analyse de la pratique mathématique doive utiliser les mêmes notions que la pratique. La théorie de la pratique mathématique peut s'éloigner radicalement parfois de la pratique, parfois même les écarts de la théorie et de la pratique sont si grands que la théorie en devient plus ou moins inutile, par exemple, la théorie des types des Principia Matbematica qui, pour contenir trop de notions ad hoc, perd tout contact avec la réalité de la pratique, alors que la théorie contemporaine de Zermelo, appelée maintenant «structure cumulative des rangs», pour la théorie axiomatique des ensembles, plus sobre conceptuellement, a été adoptée par les praticiens. La siruation est comparable à la mécanique quantique où l'interprétation indéterministe de Copenhague apparaît moins arbitraire que l'interprétation déterministe de Broglie ou de Bohm, par exemple.

Les fondements des mathématiques, dans le sens défini plus haut, ont selon moi deux versants : un versant structurel ou extensionnel, où le langage ou les langages de la pratique sont employés et élargis afin de clarifier les notions fondamentales et d'en tirer le plus de fruits possibles sur le plan formel, i.e. la logique au sens technique; l'autre versant, je l'appelle conceptuel on intensionnel; c'est l'analyse conceptuelle au sens propre où la pratique mathématique est analysée critiquement ${ }^{4}$. Ici les notions ou les concepts, les principes et les méthodes sont radicalisés, l'analyse tend à les réduire à leur plus petit noyau in-

4. L'analyse critique suppose que l'on ne se limite pas à l'utilisation d'un langage mathématique (ou logique). 
tentionnel pour faire emerger les raisons, les justifications théoriques ou théorétiques, si l'on veut mettre l'accent sur la teneur en théorie des fondements des mathématiques. Mieux, il faudrait dire fondements métathéorétiques, puisque les fondements conceptuels prennent pour objet les théories mathématiques qu'élabore la pratique mathématique.

Les attitudes fondationnelles dans cette description se résument pour moi à ce que j'appelle le structuralisme, qui regroupe des options diverses comme le réalisme, le formalisme au sens d'A. Robinson, le nominalisme, et d'autre part le constructivisme dans toutes ses variétés, parmi lesquelles l'intuitionnisme occupe une place centrale. Remarquons que le constructivisme ne recouvre pas nécessairement l'ensemble des fondements conceptuels, mais les fondements conceptuels semblent déboucher naturellement sur le constructivisme, alors que les fondements structurels invitent à une interprétation structuraliste. J'emploie structuralisme ici d'abord dans le sens de la théorie bourbakiste des structures, mais surtout dans le sens de Bernays qui définit les mathématiques comme phénoménologie théorétique ou théorique des structures ${ }^{5}$.

3. Cette systématisation ou cette unification des recherches fondationnelles n'aurait qu'une valeur esthétique, si elle ne contribuait à préciser notre analyse des mathématiques et de la logique mathématique contemporaines. La problématique fondationnelle, en mettant l'accent sur les aspects distinctifs, les variantes fondationnelles, les composantes essentielles des concepts ou des notions, peut permettre de mieux comprendre les mathématiques et par là même d'orienter la recherche dans des directions plus susceptibles de porter des fruits.

Si la théorie des ensembles est généralement considérée comme la théorie fondamentale des mathématiques, cela ne signifie aucunement qu'elle n'a pas besoin de fondements, au sens d'analyse critique des notions fondamentales. Les constructions

5. BERNAYS, P. "Comments on Wittgensteins Remarks on the Foundations of Mathematics», in Philosophy of Mathematics, ed. by Benacerraf and Putnam, Prentice Hall (Englewood Cliff, 1964), p. 528. 
de la théorie axiomatique des ensembles fournissent un bon exemple d'élaboration structurelle ou extensionnelle. Prenons l'axiomatisation de Zermelo, dite structure cumulative des rangs ou des types, qu'on définit de la façon suivante:

$\mathrm{V}_{0}=\phi \quad$ (pour l'ensemble vide) $\varphi$

$\mathrm{V}_{\alpha+1}=\mathrm{V}_{\alpha} \cup \mathrm{P}\left(\mathrm{V}_{\alpha}\right)$ pour tous les ordinaux

Remarquons que cette hiérarchie mène vers ce que Cantor appelait «eine absolut unendliche oder inkonsistente Vielheit». Mais en considérant un ordinal-limite :

$\mathrm{V}_{\alpha}=\bigcup_{\beta>\alpha} \mathrm{V}_{\beta}$

on peut obtenir un segment suffisant pour la théorie des ensembles ou la théorie de la catégorie de toutes les catégories de Lawere en prenant $\mathrm{Cat}_{\alpha}$ comme l'indique MacLane ${ }^{6}$. La structure hiérarchique a été utilisée surtout dans la théorie des modèles de la théorie des ensembles pour obtenir les résultats de consistance relative et d'indépendance. Gödel emploie cette structure ramifiée pour ses ensembles constructibles et Cohen loge ses ensembles génériques entre $V_{\alpha}$ (pour le modèle minimal ${ }^{M}$ ) et $V_{B}$ pour $\beta>\alpha$ (dans le modèle générique ${ }^{N}$ ). Cependant, la théorie pleine des ensembles est une théorie du second ordre, alors que le résultat de Cohen n'est valable que pour le premier ordre et on peut montrer que le premier ordre est dérivé du second. Le schéma de compréhension du premier ordre

$$
\begin{gathered}
\forall_{\mathrm{a}} \forall_{\mathrm{x}_{1}} \ldots \forall_{\mathrm{x}_{\mathrm{b}}} \exists_{\mathrm{x}} \forall_{\mathrm{y}}(\mathrm{y} \epsilon \mathrm{x} \leftrightarrow \\
\left.\left[\mathrm{y} \epsilon \alpha \wedge A\left(\mathrm{y}, \mathrm{x}_{1} \ldots, \mathrm{x}_{\mathrm{n}}\right)\right]\right)
\end{gathered}
$$

constitue une restriction de l'axiome du second ordre

$$
\forall_{\mathrm{a}} \forall \mathrm{X} \exists_{\mathrm{x}} \forall_{\mathrm{y}}[\mathrm{y} \in \mathrm{x} \leftrightarrow(\mathrm{y} \in \alpha \wedge \mathrm{X}(\mathrm{y}))]
$$

6. S. MACLANE, "Categorical Algebra and Set-Theoretic foundations" in Axiomatic Set Tbeory, ouvr. cit., p. 235. 
ou $\mathrm{X}$ est une variable du second ordre (c'est-à-dire que $\mathrm{X}$ exprime la propriété d'être un sous-ensemble). Dans ce sens, il est naturel de penser que l'hypothèse du continu est décidable au second ordre

$$
\left(\mathrm{ZF} \vdash_{2} \mathrm{CH}\right) \vee\left(\mathrm{ZF} \vdash_{2} \sim \mathrm{CH}\right)
$$

sous la forme

$$
\mathrm{X} \subset \mathrm{V}_{\omega+1} \rightarrow \overline{\overline{\mathrm{X}}} \leq \overline{\overline{\mathrm{V}}}_{\omega} \vee \overline{\overline{\mathrm{X}}}=\overline{\overline{\mathrm{V}}}_{\omega+1}
$$

pour $\mathrm{V}_{\omega}$ comme collection des ensembles finis et $\mathrm{X}$ comme sous-ensemble de l'ensemble des réels. La position structuraliste est représentée ici par Gödel qui, dans son article «What is Cantor's Continuum Hypothesis » affirme que

«...set-theoretical concepts and theorems describe some welldetermined reality in which Cantor's conjecture must be either true or false ».

Soutenir donc que l'hypothèse du continu est décidable au second ordre n'est autre chose que préciser le point de vue de Gödel ${ }^{7}$. Mais la valité de la hiérarchie cumulative - les rangs supérieurs - n'est pas pour autant assurée. En effet, si on peut facilement accorder l'évidence intuitive de la notion d'ensemble de, "set of », appelée notion itérative d'ensemble pour les ensembles finis, la chose est moins sûre pour les ensembles infinis. $\mathrm{La}_{\mathrm{a}}$ propriété d'être un sous-ensemble arbitraire ou un prédicat arbitraire est-elle constructible, au sens propre? Le constructivisme n'accepte pas le donné d'une structure cumulative sur les ensembles infinis ; il faut plutôt penser en termes de procès itératif infini ou plutôt effini, c'est-à-dire en dehors du fini, au-delà du champ du fini. Selon moi, c'est du côté du quantificateur universel qu'il faut regarder - vers son imprédicativité, comme disaient les premiers constructivistes. On se souvient de la définition de la notion d'ensemble que l'on trouve chez Cantor «un ensemble est une

7. Ceci répond à une objection soulevée par A. MOSTOWSKI, « Recent Results in Set Theory (Discussion) 》 in Problems in the Pbilosopby of Matbematics, North-Holland (Amsterdam : 1967), p. 107. 
synthèse ou un rassemblement (《Zusammenfassung 》) d'objets distincts déterminés de notre intuition ou de notre pensée (les «éléments » de l'ensemble) en un tout ( $\mathrm{Zu}$ einem Ganzen») ${ }^{8}$. C'est ici que «tous», le quantificateur universel devient un tout, une totalité. Ce passage de tous à tout a une longue histoire philosophique. Le monde ou l'univers comme tout comprend toutes choses. Mais le monde, le tout, le «to pan» des Grecs, est-il autre chose qu'une unité idéale, qu'un concept sans référent? L'idée de tout implique celle de contenant: le tout, par exemple, $\chi^{\prime}{ }_{0}$. contient tous les entiers, $\chi^{\prime}{ }_{1}$. tous les réels, $\chi^{\prime}{ }_{2}$, toutes les fonctions sur les réels, ainsi de suite. Le contenant, le tout, même lorsqu'on le suppose infini, est cependant représenté comme fini, puisqu'il est lui-même contenu dans d'autres contenants de la hiérarchie. Comment se représente un contenant contenu si ce n'est comme fini? Cette idée du contenant universel à une longue tradition de la sphère de Parménide à l'univers sphérique d'Einstein. Le continu arithmétique signifie une constructibilité indéfinie alors que le continu ensembliste signifie un tout achevé, une totalité infinie contenue dans un ensemble. L'ensemble infini est donc considéré comme la totalité des éléments «dans» l'ensemble.

Cette critique du quantificateur universel et des ensembles infinis que je viens d'esquisser s'applique particulièrement bien aux axiomes forts d'infinité, par exemple les axiomes d'inaccessibilité et de mesurabilité ${ }^{9}$ On sait qu'un cardinal est inaccessible lorsque 1) pour $B$ plus petit que $A, A$ (transfini), A n'est pas la somme d'un nombre $B$ de cardinaux plus petits que $A$ et 2 ). A est plus grand que l'ensemble des sous-ensembles de B :

1) $A>\omega$

2) $\forall_{\mathrm{x}} \subset \mathrm{A}$, Card. $(\mathrm{x})<\overline{\overline{\mathrm{A}}} \rightarrow \cup_{\mathrm{n}}(\mathrm{x})<\overline{\overline{\mathrm{A}}}$

3) $\forall \mathrm{B}, \mathrm{B}<\mathrm{A} \rightarrow 2^{\mathrm{B}}<\mathrm{A}$

8. CANTOR, G, «Gesammelie Abhandlungen, hrsg. von E. Zermelo, Georg Olms (Hildesheim : 1966), p. 282.

9. Pour la définition de ces notions, voir J.R. SHOENFIELD, Mathematical Logic, Addison-Wesley (Reading, Mass. : 1967). 
Pour un cardinal mesurable, on a un idéal sur un ensemble transfini qui partitionne les sous-ensembles de l'ensemble transfini en petits et grands sous-ensembles.

Pour A ensemble transfini, ou a un sous-ensemble $B$ de l'ensemble des parties ou des sous-ensembles $\mathrm{P}$ (A) tel que

a) pour tout sous-ensemble $\mathrm{C}$ de $\mathrm{B}$ tel que $\overline{\overline{\mathrm{C}}}<\overline{\overline{\mathrm{A}}}$, $\cup \mathrm{C} \in \mathrm{B}$

b) pour tout sousensemble $C$ de $A, C \in B \vee A-C \in B$

c) $\cup B=A$

d) $A \notin B$

Remarquons que l'axiome de mesurabilité fait appel à une définition du troisième ordre, puisque l'idéal est défini sur la totalité des sous-ensembles d'un ensemble transfini. Ici la représentation du contenant est claire: deux sous-contenants d'un contenant principal déterminé. De plus, on suppose que le cardinal du contenant principal est transfini. J'appelle «principe ou procès transarithmétique ${ }^{10}$, la procédure qui consiste à définir, «à contenir » le procès arithmétique dans les limites d'un procès cumulatif pour ensuite l'appliquer sur l'accumulation des contenants infinis, qui eux-mêmes sont constitués par la négation du procès arithmétique.

4. Je veux donner un dernier exemple d'analyse critique pour la théorie axiomatique des ensembles, le schème de réflexion. Je prends la formulation de Krivine: on a une relation fonctionnelle $\mathrm{y}=\mathrm{W}_{\alpha}$ dont le domaine est l'ensemble des ordinaux $\mathrm{O}_{\mathrm{n}}$ croissante, i.e. $\alpha \leq \beta \rightarrow \mathrm{W}_{\alpha} \subset \mathrm{W}_{\beta}$ et continue (si $\alpha$ est un ordinal limite, $W_{\alpha}=\cup W_{\beta}$ ) considércns $W$ comme la collection qui est l'union des ensembles $W_{\alpha}$,

$$
W_{(\mathrm{x})}: \exists_{\alpha}\left[O_{\mathrm{n}}(\alpha) \wedge \mathrm{x} \in \mathrm{W}_{\alpha}\right]
$$

10. Voir Yvon GAUTHIER, «Logique mathématique et philosophie des mathématiques», Dialogue, vol. X (1971, no. 2, pp. 243-275, en part, pp. 272-273). 
Si $E\left(x_{1}, \ldots ., x_{n}\right)$ est un énoncé sans paramètres, rous avons ce qui suit

$$
\begin{aligned}
& \forall_{\alpha} \exists \beta>\alpha \forall_{\mathrm{x}_{1}} \ldots \forall_{\mathrm{x}_{\mathrm{k}}}\left[\mathrm{x}_{1} \in \mathrm{W}_{\beta} \wedge \ldots \wedge \mathrm{x}_{\mathrm{k}} \in \mathrm{W}_{\beta}\right. \\
& \left.\rightarrow\left(\mathrm{E}^{\mathrm{W}}\left(\mathrm{x}_{1}, \ldots, \mathrm{x}_{\mathrm{k}}\right) \leftrightarrow \mathrm{E}^{\mathrm{W}_{\beta}}\left(\mathrm{x}_{1}, \ldots, \mathrm{x}_{\mathrm{k}}\right)\right)\right]
\end{aligned}
$$

Ce qui signifie que l'énoncé $\mathrm{E}^{\mathrm{w}_{\beta}}$ est équivalent à l'énoncé $\mathrm{E}^{\mathrm{W}}$ pour la simple raison qu'il appartient à la hiérarchie des ordinaux (comme ordinal limite). Ainsi l'univers ou le contenant universel se réfléchit-il dans un de ses sous-contenants; si nous nous représentons le contenant universel comme un cercle ou une sphère et la structure cumulative des rangs comme une pyramide renversée, on peut réfléchir de la façon suivante sur les réflexions: la hauteur ou le niveau dans la structure cumulative correspond à son rang ordinal et la largeur du niveau correspond à son cardinal ${ }^{11}$.

On a la structure cumulative des rangs

$\mathrm{V}_{0}=\phi$

$\mathrm{V}_{\alpha+1}=\mathrm{V}_{\alpha} \cup \mathrm{P}\left(\mathrm{V}_{\alpha}\right)$ pour tout ordinal

$V_{\alpha}=\underset{\beta<\alpha}{\cup} V_{\beta}$ pour tout ordinal limite

et nous nous représentons l'univers de la façon suivante:

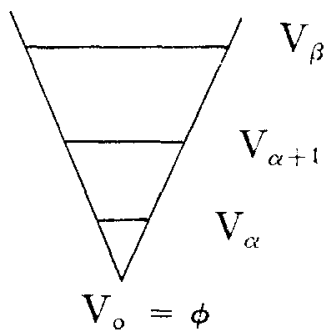

pour $\mathrm{V}=\mathrm{V}_{\beta}$ (voir appendice). Mais cet univers ne représente qu'une ordination, i.e. chaque ensemble et chacun de leurs éléments ne sont ordonnés qu'une fois. $\mathrm{Si}$ nous plongeons cette structure cumulative dans un univers plein, c'est-à-dire dans une sphère de cardinalité $\mathrm{R}_{\gamma}$, on obtient alors une ordination

11. J.L. KRIVINE, Théorie axiomatique des ensembles, P.U.F. (PARIS : 1969), PP. 64-72. 
transfinie des ensembles et de leurs éléments, chaque point de la circonférence de la sphère ou du cercle constituant la base d'une structure cumulative ou son ensemble vide $\phi$. Chaque ensemble comprenant l'ensemble des sous-ensembles qui le précède dans la hiérarchie, on a $\mathrm{V}_{\alpha+1}=\mathrm{P}\left(\mathrm{V}_{\alpha}\right)$ et $\mathrm{V}_{\omega+1}=\mathrm{P}\left(\mathrm{V}_{\omega}\right)$. Mais cette hiérarchie doit elle-même être hiérarchisée transfiniment dans un univers plein, i.e. dans une sphère de cardinalité $?^{\prime}{ }_{1}$

Cette ordination transfinie qui donne $\chi_{y}^{\prime}$, et que nous notons $\mathrm{R}(\omega)$, nous l'appelons réflexion coordinale universelle: en effet, dans cette construction, chaque élément, par ordination transfinie, réfléchit tous les autres éléments en même temps que l'univers tout entier; chaque élément est un miroir à facettes de cardinal $\lambda^{\prime}$,

De cette façon, l'hypothèse du continu est violée ou falsifiée, puisque le cardinal du continu est ici $\chi_{2}^{\prime}$, ou tout autre cardinal transfini $\chi^{\prime}$ Soulignons que notre notion d'ordination est différente de la notion d'ordre ou de bon ordre - c'est, en fait, une multiplicité de puissance $Z^{\prime}$ y de bons ordres sur tous les éléments de l'univers. Remarquons aussi que notre construction ne donne pas, comme on pourrait le penser, l'ensemble des fonctions sur le continu (qui donnerait $\ell_{2}^{\prime}$, ou un autre cardinal plus grand) simplement parce que le continu est constitué par l'ensemble des réflexions $\mathrm{R}(\omega)$. La justification d'une telle construction par rapport à la structure cumulative, c'est que la structure cumulative représente une construction finie incomplète (procédant par étapes). Après coup, on peut y voir une lointaine analogie avec le principe philosophique de Leibniz, selon lequel les monades reflètent l'univers entier et toutes les autres monades ou encore le principe du tout en tout d'Anaxagore.

5. Je veux passer maintenant de l'univers plein à l'univers raréfié de la théorie intuitioniste du sujet créateur. L'idée du sujet créateur a été introduite en 1948 par Brouwer pour justifier ses contre-exemples de la forme:

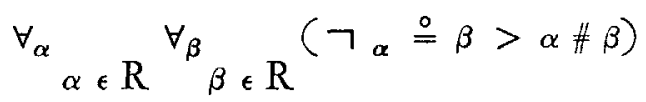

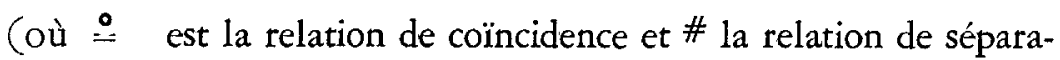
tion pour les nombres réels) et a connu depuis quelques années 
un renouveau important dans les travaux de Kreisel, Myhill et Troelstra sur l'intuitionnisme ${ }^{12}$. Le concept central de l'intuitionnisme est celui de procès, selon moi. Tous les autres concepts dérivent de celui-là : déploiements (spreads), espèces, séquences ou séries irrégulières (lawless), séquences de choix, séquences constructives et sujet créateur ou constructeur. La notion de procès mathématique implique pour moi celle de sujet dans la mesure où tout procès conscient renvoie à un agent. Par exemple, pour revenir au principe de réflexion; la théorie des démonstrations comporte aussi des principes de réflexion; cependant ces derniers ne réfléchissent pas l'univers dans un de ses sous-univers, mais plutôt la convertibilité, pour le sujet pensant, de la prouvabilité en validité ; ainsi $\forall_{\mathrm{P}}\left[\operatorname{Prouv}_{\mathrm{s}}(\mathrm{p}\right.$, " " $\varphi$ ") $\rightarrow \varphi]$ où $\varphi$ est $\mathrm{u}$ n terme dont la valeur est le nombre de Gödel pour $\varphi$.

C'est sans doute là l'une des raisons qui font dire à Kreisel que la théorie des démonstrations comme théorie des notions et des constructions n'existe pas encore, et aussi que la théorie des fonctions récursives s'arrête là où commence la théorie des démonstrations ${ }^{13}$. Mais du moins voit-on que le principe de réflexion dont il s'agit ici a peu en commun avec le principe de réflexion dans l'univers ensembliste.

Les mathématiques étant une activité constructive de l'esprit, il faut un constructeur ou un «structurateur» pour les structures mathématiques. Mais il ne faut pas croire que cela signifie l'introduction de la psychologie en mathématiques. Tout ce dont nous avons besoin, c'est d'une légitimation pour le procès mathématique, qui ne requiert qu'un sujet impersonnel, transcendantal, comme dit Husserl (ou encore un agent purement linguistique).

12. Voir J. MYHILL, «Formal Systems of Intuitionistic Analysis I » in Logic, Metbodology and Pbilosopby of Science III, ed. by van Rootselaar and Staal, North-Holland (Amsterdam: 1968), pp. 161-162. Voir aussi, pour un résumé des problèmes de l'intuitionnisme et de sa formalisation, $\mathrm{J}$. Myhill «The formalization of intuitionism》 in lat pbilosopbie contemporaine, vol. I. ed. R. Klibansky, (Firenze, 1968), pp. 324-341.

13. G. KREISEL, «Some reasons for generalizing recursion theory 》 in Logic Colloquium '69, ed by Gandy and Yates, North-Holland (Amsterdam : $1971)$, p. 162. 
Les axiomes pour le sujet constructeur ont été introduits par Kreisel et repris par Troelstra ${ }^{14}$.

$\left(\Sigma \vdash_{M} \mathrm{~A}\right)$ v $\neg\left(\Sigma \vdash_{M} \mathrm{~A}\right)$

$A \rightarrow \Lambda \Sigma\left(\neg \neg V_{M}\left(\Sigma \vdash_{M} A\right)\right)$

$\mathrm{V} \approx \mathrm{V}_{\mathrm{M}}\left(\Sigma \vdash_{\mathrm{M}} \mathrm{A}\right) \leftrightarrow \mathrm{A}$

$(\vee$ et $\wedge$ pour $\exists$ et $\forall)$

ce qui signifie que le sujet constructeur a une preuve de $A$ à l'étape $M$ ou il n'en a pas et il existe une preuve de $A$, elle ne peut pas être inaccessible et il suffit qu'un sujet créateur ait une preuve de $A$ à une étape donnée pour qu'existe une preuve de A. Avant d'aborder notre critique de ces axiomes, voyons ce qu'on peut en tirer. En premier lieu, le schème de Kripke

$V_{\chi}[(\Lambda \times(\chi x=0) \leftrightarrow \neg A$

$\left.\wedge\left(V_{x}(x x \neq 0) \rightarrow A\right)\right]$

ou dans sa forme forte

$V_{x}\left(V_{x}(\chi x \neq 0) \leftrightarrow A\right)$

qui signifie qu'il suffit qu'existe une séquence réalisable (une séquence étant un procès qui associe à tout nombre naturel ou objet mathématique appartenant à une espèce donnée, ou ayant une certaine propriété) pour $\mathrm{x}$, un nombre naturel, pour qu'on ait une preuve de $A$.

$\left(r_{\mathrm{N}} \mathrm{A}\right) \rightarrow x \mathrm{n}=1$

$\left(\neg \vdash_{\mathrm{N}} \mathrm{A}\right) \rightarrow x \mathrm{n}=0$

Une séquence définie de cette façon est appelée empirique; une séquence est mathématique, lorsqu'il n'est pas nécessaire de la définir par étapes, par exemple, une fonction récursive primitive. Il y a plusieurs problèmes liés à cette axiomatisation du sujet créateur, tels ceux de la continuité et de la succession chronolo-

14. A.S. TROELSTRA, "The theory of Choice Sequences》 in Logic, Metbodology and Pbilosopby of Science III, ouvr. cit., p. 212. 
gique des étapes. Ces problèmes sont centrés autour de la notion de séquence de choix.

6. Toute centrale qu'elle soit, la notion de séquence de choix a fait l'objet de définitions diverses. J'en propose une ici et je lui donne un nouveau nom: séquence polymorphe ou polygénétique. Une séquence de choix est en effet une séquence engendrée par des choix sucessifs de valeurs et des limitations qui leurs sont imposées. On commence par choisir les valeurs. $\mathrm{x}_{0}, \mathrm{x}_{1}, \ldots$ 》 pour une séquence de choix $\alpha$, puis on décide à un certain moment de limiter les choix futurs de valeurs en imposant que $\beta=\Gamma_{0} \alpha_{0}$ où $\alpha \quad$ est une nouvelle genèse de choix de valeurs et $\Gamma_{0}$ une opération continue (une fonctionnelle) sur la séquence $\alpha$ : en d'autres mots, sur un segment initial de valeurs de $\alpha$, on peut ajouter l'information $\alpha=\mathrm{e} / \beta$, poursuivre le choix de valeur sur $\beta$ et ainsi de suite indéfiniment. Kreisel et Troelstra dans « Formal systems for some branches of intuitionistic analysis » (Annals of Mathematical Logic, vol. 1, no. 5) parlent, pour leur part, d'un principe de données analytiques formulé de la façon suivante:

$$
\mathrm{A}(\alpha) \rightarrow \mathrm{V}_{\mathrm{c}}[\alpha \in \mathrm{e} \wedge \Lambda \beta \mathrm{A}(\mathrm{e} / \beta)]
$$

pour $\alpha$ et $\beta$ comme séquences de choix, $\mathrm{A}$ une propriété et $\mathrm{e}$ un ensemble analytique (sur l'espace de Baire de toutes les séquences de choix). On voit que ce qui est requis ainsi, c'est que le sujet constructeur assiste aussi loin que possible à la genèse de ses propres constructions mathématiques.

Dans ce qui suit, je veux tenter de répondre brièvement à une question posée par Kreisel et Troelstra (un problème ouvert de l'intuitionnisme) ${ }^{15}$. Comment relier les étapes (stages) de la théorie des séquences de choix et séquences irrégulières aux «étapes» de la théorie du sujet créateur? J'ai tenté ailleurs de formuler une théorie axiomatique des fondements à l'aide de quatre notions fondamentales, celles de stase, de procès, de fini et d'effini

15. Voir A.S. TROELSTRA, Principles of Intuitionism, Lecture Notes in Mathematics, Springer (Berlin - Heidelberg - New-York : 1969), p. 107. 
(sans rapport avec la notion de métafini en théorie de la métarécursion) ${ }^{16}$. Je veux encore une fois être bref.

J'appelle «domaine dominable» l'activité constructive en général et «domaine dominé » le domaine des constructions finies. Le domaine dominé est celui de l'arithmétique finie, des lois logiques élémentaires (intuitionnistes). Le domaine dominable est celui des itérations effinies, i.e. hors du fini, donc par exemple, les fonctions récursives primitives, les séquences de choix, e.g., la suite des nombres naturels qui est effinie pour moi à la différence des intuitionnistes qui acceptent l'ensemble des nombres naturels comme un donné. Les structures nouvelles que l'on admet, plutôt que d'être définies par un procès cumulatif, sont engendrées par un processus d'expansion du domaine. Le point de vue ici, n'est plus celui de l'arithmétique ordinale, mais celui de la géométrie projective finie. Le procès expansif ne comporte pas de niveaux, mais des degrés (au sens géométrique d'ouverture d'un angle) ou des périodes qu'on peut exprimer axiomatiquement de la façon suivante :

$\left(\Sigma \vdash_{\mathrm{D}(\alpha)} \mathrm{A}\right) \vee \neg\left(\Sigma \vdash_{\mathrm{D}(\alpha)} \mathrm{A}\right)$

$A \rightarrow \Lambda \Sigma\left(\neg \neg V_{D^{\prime}(\alpha)}\left(\Sigma \vdash_{D(\alpha)} A\right)\right)$

$\mathrm{V} \Sigma \mathrm{V}_{\mathrm{D}(\boldsymbol{\alpha})}\left(\Sigma \mathrm{V}_{\mathrm{D}(\boldsymbol{\alpha})} \mathrm{A}\right) \leftrightarrow A$

Les degrés $\alpha^{\prime}, \alpha^{\prime \prime}, \alpha^{\prime \prime \prime}$ représentent ainsi un procès expansif qui vise un horizon constructif effini: l'horizon n'est jamais accessible, mais est toujours présent. J'axiomatise la notion d'horizon $\mathrm{H}$ de la façon suivante:

$$
\left(\Lambda \Sigma \Lambda \mathrm{A} \vdash_{\mathrm{D}} \mathrm{A} \subset \mathrm{H}\right) \wedge \neg\left(\Lambda \Sigma \Lambda \mathrm{A} \vdash_{\mathrm{D}} \mathrm{A} \supset \mathrm{H}\right)
$$

J'évite ainsi toute théorie hiérarchique ou stratificationnelle de types, de rangs ou de niveaux. L'horizon est indéfiniment ou effiniment expansible, mais il indique les limites de la visualisation ${ }^{17}$. L'ordre des étapes est remplacé par une progression

16. Dans un ouvrage sur les fondements des mathématiques à paraître bientôt.

17. Le modèle du champ visuel donné ici doit être complété par un modèle du champ linguistique, où la performance du sujet parlant doit être com- 
vers l'horizon constructif qui détermine aussi les genèses successives des séquences de choix et aussi bien les monogenèses des fonctions récursives primitives par expansions périodiques. Je pense ici que l'idée de procès générateur d'expansions et de périodes ou degrés sur un horizon rend fidèlement l'interprétation constructiviste exigée par Kreisel et Troelstra.

7. Je voudrais dire quelques mots, avant de passer aux conclusions sur l'interprétation structurelle du constructivisme. Je pense surtout à l'interprétation de Vesley, qui dans son travail «A palatable substitute for Kripke's schema » a proposé le schème suivant ${ }^{18}$ :

Dense $(\neg \mathrm{A}(\alpha), v) \& \forall \alpha(\neg \mathrm{A}(\alpha) \supset$

$\exists \beta \mathrm{B}(\alpha, \beta)) \supset \forall \alpha \exists \beta(\neg \mathrm{A}(\alpha) \supset \mathrm{B}(\alpha, \beta))$

qui signifie qu'à toute séquence de choix $\alpha$ dans l'espèce dense négative $\mathrm{A}$ correspond une séquence de choix $\mathrm{B}$ sur l'espèce universelle $v$. Ici, l'interprétation structurelle ou objectiviste est fondée sur le principe des extensions continues des fonctions continues de l'analyse classique. Mais c'est sans doute là déroger à la nature des mathématiques intuitionnistes qui cherchent à reconstruire les mathématiques classiques en utilisant des méthodes constructivistes.

Il n'est pas surprenant alors que Joan Rand Moschovakis, dans un article récent dans le Journal of Symbolic Logic «Can there be no non-recursive functions? $\gg^{19}$, ait pu démontrer la con-

prise en fonction de sa compétence, i.e. sa connaissance du champ linguistique. Pour nous il n'y a pas de pensée sans langage, ou plutôt, le langage est la pensée. Il faudrait donc déterminer quelles sont les opérations linguistiques qui correspondent aux opérations mathématiques, en d'autres mots, quels sont les rapports de la syntaxe mathématique et de la syntaxe des langues naturelles. C'est cette conjonction de la visualisation et de la verbalisation qui constitue pour nous l'assise dernière du sujet pensant. Mais c'est toute une théorie philosophique qu'il faudrait élaborer ici.

18. R.E. VESLEY «A palatable substitute for Kripke's schema 》 in Instuitionism and Proof Theory, ed. by Myhill, Kino, Vesley, North-Holland Amsterdam : 1970, pp. 197-207.

19. J.R. MOSCHOVAKIS, «Can there be no nonrecursive functions 》? in The Journal of Symbolic Logic, vol. 36, number 2, pp. 309-315. 
sistance de la thèse de Church - qui, comme on le sait, affirme que toute fonction effectivement computable est récursive générale - avec le système intuitionniste de Kleene pour l'analyse exposé dans son ouvrage écrit en collaboration avec Vesley The Foundations of Intuitionistic Matbematics ${ }^{20}$.

D'autre part, Dana Scott a donné une interprétation structurelle topologique - prenant pour modèle le treillis des sousensembles ouverts d'un espace topologique donné - pour laquelle le schème de Kripke est consistant ${ }^{21}$. Or, on sait que le schème de Kripke contredit la thèse de Church, puisque les fonctions constructives au sens intuitionniste débordent le domaine des fonctions effectivement computables ${ }^{22}$. Cet exemple montre peut-être la valeur toute relative des résultats formels de consistance relative et d'indépendance pour l'intuitionnisme aussi bien que pour la théorie des ensembles. Mais il montre aussi que l'approche structurelle en logique mathématique et dans les fondements des mathématiques, pour être riche et féconde, ne suffit sans doute pas pour résoudre les problèmes ouverts ou pour découvrir des axiomes neufs justifiables, réclamés par Gödel en particulier. La rigueur informelle, dont parle Kreisel, consiste peut-être à prendre ses distances par rapport à l'approche structurelle (qui, elle-même, comprend l'approche formelle technique) afin de laisser libre champ à l'intuition et à l'analyse conceptuelle. Mais là encore, c'est une dialectique de la complémentarité qui joue et je pense que cette dialectique est essentielle à la recherche fondationnelle en logique et en mathématiques.

20. S.C. KLEENE and R.E. VESLEY, The Foundations of Intuitionistic Matbematics, North-Holland, Amsterdam, 1965.

21. D. SCOTT \& Extending the topological interpretation to intuitionistic analysis $»$ in Logic and Foundations of Matbematics, ded. to Prof. A Heyting on his 70th birthday, Wolter-Nordhof, Groningen: 1968, pp. 194-210 et «Extending the topological interpretation to intuitionistic analysis II $»$ in Intuitionism and Proof Theory, ouvr. cit., pp. 235-255; voir aussi la discussion de Kreisel «Chruch's thesis : a kind of reducibility axiom for constructive mathematics» dans le même volume, pp. 121-150, en part, pp. 128 et 137.

22. Voir l'important article de KREISEL, «Lawless sequences of natural numbers $\gg$ in Logic and Foundations of Matbematics, ouvr. cit., pp. 222-248, où la notion de fonction constructive est prise comme notion primitive non-équivalente à la notion de fonction récursive. 
8. Il est temps de conclure. Je voudrais d'abord dire quelques mots concernant la théorie des catégories. Ce que j'en ai dit ne couvre que les travaux de MacLane et de Lawvere avant la théorie des topoi. Feferman ${ }^{23}$ a montré comment on pouvait formaliser la théorie des catégories à l'aide du schème de réflexion dans la théorie des ensembles et MacLane a admis la possibilité de formuler la même théorie sur la structure cumulative des rangs. Ce que je sais de la théorie des topoi m'incline à croire que c'est essentiellement une théorie structurelle, géométrico-algébrique, engendrée par le couple géométrie algébrique (théorie des faisceaux) et algèbre homologique (théorie des catégories) . Il semble bien cependant que ce point de vue, comme le point de vue logico-algébrique, qui connaît une croissance structurelle accélérée dans sa variété cylindrique, ne sera pas moins fécond pour la recherche fondationnelle. Si l'on considère que les recherches fondationnelles et la logique mathématique ont été dominées par les courants de l'arithmétisation (liée à la théorie des démonstrations), de l'algébraïsation (correspondant à la théorie des modèles), la tendance géométrisante pourrait bien se faire une place. Mais si la géométrie a touiours eu une vocation fondationnelle constructiviste, elle a sans doute renié partiellement son caractère constructiviste en s'algébraïsant. Mais il n'y a là aucune condamnation. L'intérêt mathématique d'une théorie est parfois inversement proportionnel à son intérêt fondationnel. Cela ne veut pas dire qu'il faille identifier fondationnel à nonmathématique ou encore philosophique. Loin de là. Comme je l'ai indiqué au début, les fondements des mathématiques ne sont jamais réductibles à la philosophie des mathématiques, même si certaines considérations philosophiques peuvent ou doivent entrer en ligne de compte.

Un autre aspect que je n'ai pas touché et qui est sans doute important pour la problématique fondationnelle, c'est le probì̀me des relations entre la physique et les mathématiques. J'ai abordé ce problème dans un article récent où j’ai soutenu l'idée

23. S. FEFERMAN, "Set-theoretical foundations for category theory. With an appendix by Georg Kreisel 》 in Reports of the Midwest Category Seminar III, ed by S. Maclane. Lecture Notes in Mathematics, Springer (Berlin - Heidelberg - New-York: 1969), pp. 201-247. 
que la physique et les mathématiques avaient ultimement le même objet: la construction du continu ou, ce qui est la même chose, du discontinu ${ }^{24}$. Hermann Weyl, von Neumann, parmi d'autres, soutenaient que seules les mathématiques applicables à la physique (et par extension aux autres sciences) pouvaient être légitimées, c'est-à-dire possédaient un intérêt fondationnel. Mais il est sans doute impossible de faire la démarcation entre les mathématiques qui sont applicables et celles qui ne le sont pas. L'exemple des fondements de la mécanique quantique est instructif à cet égard. Une grande variété de structures logiques et logico-algébriques sont étudiées dans ce qu'on est convenu d'appeler la logique quantique - logiques multivalentes, treillis, etc... même la théorie des ensembles n'a plus la pureté (si l'on peut encore utiliser ce mot) d'une théorie inutile pour la physique.

Quoique que je me qualifie moi-même de constructiviste, mon propos n'a pas été d'exposer les mérites du constructivisme aux dépens de ce que j'ai proposé d'appeler le structuralisme, plutôt que non-constructivisme. J'ai essayé au contraire de montrer les mérites respectifs des deux approches.

Il est inutile, selon moi, de tenter de réduire l'importance des recherches purement formelles, comme il est vain de rejeter les recherches fondationnelles qui seraient ou trop philosophiques ou n'auraient qu'une valeur heuristique. Je pense qu'on ne peut pas imposer de bornes a priori à l'invention ou à l'imagination mathématique, pas plus qu'on ne peut tracer la ligne de partage entre philosophie et mathématiques. Mais je l'ai dit au début, la division du travail dans ce domaine n'a plus beaucoup de sens et le travail fondationnaliste, le fait qui le peut.

On pourrait aussi objecter que les mathématiques n'ont pas besoin d'autres fondements qu'elles-mêmes, ce qui reviendrait à dire que les mathématiques occupent tout l'horizon théorétique, ce qu'il faudrait d'abord montrer, ou, à l'inverse, qu'elles n'ont aucune visée théorétique, ce qu'il faudrait aussi montrer. Le sujet

24. Voir Y. GAUTHIER, \& The use of the axiomatic method in Quantum Physics $\gg$ in Pbilosopby of Science, vol. 38, number 3 (Sept. 1971), pp. 429.437 et « Le constructivisme et la structure des théories physiques 》 à paraître. 
même des fondements des mathématiques en fait une entreprise théorique ambivalente, si ce n'est ambiguë. Sa fonction de synthèse et d'unification la force à réunir des résultats parfois disparates, à joindre des méthodes opposées, à lier des intentions divergentes. Mais il se peut que les recherches fondationnelles en mathématiques soient un symptôme d'une nouvelle ouverture d'esprit, d'une nouvelle perspective sur le savoir qui n'est plus confiné dans des disciplines étroites. Les recherches fondationnelles, en mathématiques comme dans les autres branches du savoir, sont peut-être les nouvelles avenues de l'interdisciplinarité. Il ne fait pas de doute que la logique, par exemple, a une vocation interdisciplinaire. Quoi qu'il en soit, le chercheur fondationnaliste, le fondationnaliste, pour employer un néologisme, épistémologue qu'il faudrait appeler épistémologicien, a une double figure; il est à la fois girouette et anémomètre: girouette parce qu'il est battu par les vents de la connaissance, anémomètre parce qu'il mesure la vitesse de progression du savoir au confluent des disciplines. Et le fondationnaliste ou l'épistémologicien n'a pas de visage pour avoir ces deux masques. 


\section{FONDEMENTS DES MATHEMATIOUES}

\section{CONCEPTUELS}

1. Analyse conceptuelle (ou critique), Rigueur informelle.

2. Théorie des ensembles (la notion de structure cumulative des rangs) et fondements de la théorie des ensembles (la notion d'ensemble et ses axiomes ).

3. Concepts constructivistes (concepts combinatoires, rt fondements de la théorie des fonctions récursives et métarécurisives, fonctionnelles récursives, computabilité et calculabilité). Concepts intuitionnistes.

4. Concepts métamathématiques (théorie des démonstrations comme théorie de la réflexion).

5. Philosophie des mathématiques et philosophie mathématique.

Fondements métathéorétiques (Constructivisme)

\section{RECHERCHES} FONDATIONNELLES

Les fondements structurels utilisent les structures logiques et mathématiques comme moyens de développer langages et théories logiques et mathématiques.

Les fondements conceptucls analysent les principes et les concepts correspondant; afin de trouver une justification (une certification) pour les théories logiques et mathématiques et aussi de les réduire à une théorie fondamentale unificatrice. Les approches diverses en fondements des mathématiques peuvent ainsi être réduites à la dichotomie fondamentale constructivisme - structuralisme.

\section{STRUCTURELS}

\begin{tabular}{|c|c|c|}
\hline $\begin{array}{l}\text { Théorie des } \\
\text { ensembles } \\
\text { Transfinis }\end{array}$ & $\begin{array}{l}\text { Logique } \\
\text { Mathématique }\end{array}$ & $\begin{array}{l}\text { Théorie } \\
\text { Catégories \& } \\
\text { Théorie des } \\
\text { Topoi }\end{array}$ \\
\hline $\begin{array}{l}\text { Théorie } \\
\text { des } \\
\text { Modèles }\end{array}$ & $\begin{array}{l}\text { Théorie } \\
\text { de la } \\
\text { Récursion } \\
\text { et Méta } \\
\text { Récursion }\end{array}$ & $\begin{array}{l}\text { Théorie } \\
\text { des } \\
\text { Démonstrations }\end{array}$ \\
\hline $\begin{array}{l}\text { Structures } \\
\text { Algébriques } \\
\text { et Topologiques } \\
\text { (A. booleenne) } \\
\text { Logique } \\
\text { Algébrique } \\
\text { (Polyadique } \\
\text { Treillis, } \\
\text { Théorie des } \\
\text { ensembles } \\
\text { (cardinaux) } \\
\text { Langages } \\
\text { infinitaires. }\end{array}$ & $\begin{array}{l}\text { Structures } \\
\text { Algébriques } \\
\text { et ensemblis- } \\
\text { tes, topologi- } \\
\text { ques et Arith- } \\
\text { métiques. } \\
\text { Degrés Hiérar- } \\
\text { chiques. Théorie } \\
\text { descriptive des } \\
\text { ensembles } \\
\text { Analytiques et } \\
\text { projectifs. }\end{array}$ & $\begin{array}{l}\text { Sous-systèmes } \\
\text { de l'Analyse } \\
\text { Classique } \\
\text { Structures } \\
\text { Arithmétiques } \\
\text { (Ordinaux) } \\
\text { Définitions } \\
\text { inductives } \\
\text { Progressions } \\
\text { autonomes } \\
\text { Hiérarchie } \\
\text { Ordinale } \\
\text { Formules } \\
\text { infinies et } \\
\text { Systèmes } \\
\text { Formels }\end{array}$ \\
\hline
\end{tabular}

Fondements théorétiques (Structuralisme) 


\section{APPENDICE}

L'idée, ce n'est encore qu'une idée, qu'un univers ensembliste pleinement réfléchi - au sens de la structure cumulative des rangs et du schème axiomatique de réflexion -- viole l'hypothèse du continu, peut-être approchée ou «approximée » de façon différente (en évitant la procédure intuitive, trop intuitive peut-être, d'immersion dans une sphère de la cardinalité du continu) .

Chaque rang de la structure cumulative contient tous les ensembles des rangs précédents, nous appelons ce procès réflexion « ascendante » en analogie avec la dialectique ascendante de Platon «synagogè » ou le théorème de Löwenheim-Skolem «upward», puisque tout ensemble de rang inférieur est réfléchi à tous les rangs supérieurs. L'autre procès de réflexion, par lequel l'univers se réfléchit dans un rang donné correspondant à un ordinal-limite, je l'appelle réflexion «descendante», encore une fois en analogie avec la dialectique «descendante» de Platon «diairèsis» ou le théorème de Löwenheim-Skolem «downwatd».

Le continu combine ces deux réflexions; d'un côté, tout segment de droite est une droite, de l'autre, tout segment a la cardinalité de la droite elle-même.

La hiérarchie est donc doublement réflexive. On peut représenter de la façon suivante cette réflexion symétrique.

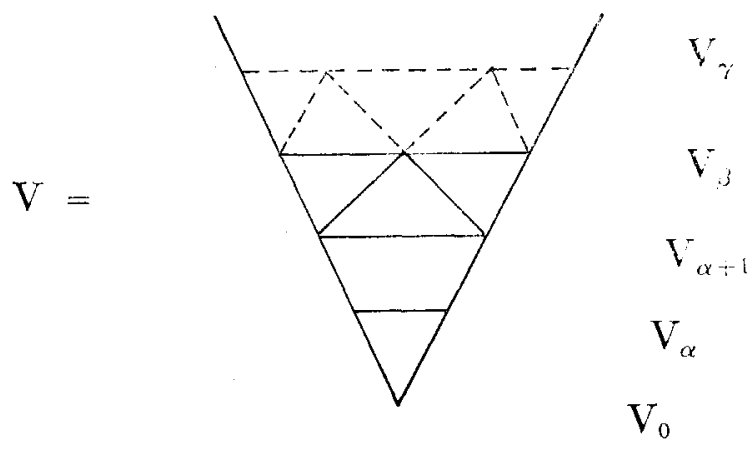

Le segment $V_{\beta}$ de la hiérarchie reflète l'univers $V$, mais l'ensemble déterminé par l'ordinal $\mathrm{B}$ réfléchit autant de copies possibles 
de lui-même dans la hiérarchie transfinie des ensembles et chaque ensemble réfléchit ou se réfléchit aussi transfiniment; si on limite le segment à $V_{\omega}$, alors les réfléxions $R(\omega)$ donnent $\chi^{\prime} \omega$ Axiomatiquement, nous avons un modèle $\nabla$ (le modèle «del $»)$ * pour la théorie des ensembles de Zermelo-Fraenkel. $\nabla$ n'est pas dénombrable, c'est un modèle pour un langage du second ordre avec cardinalité $\imath^{\prime}$. Nous exprimons le principe de réflexion coordinale ou symétrique universelle de la façon suivante:

$$
\begin{aligned}
& \forall{ }_{\alpha} \exists \circ \ell . \beta>\alpha \exists \gamma>\beta \wedge_{\alpha}, \beta, \gamma \in \mathrm{V} \forall \mathrm{X}[\{\mathrm{X} \epsilon \mathrm{V} \beta \rightarrow \\
& \left.\left(\varphi(\mathrm{X}) \leftrightarrow \varphi^{v} \beta(\mathrm{X})\right)\right\} \rightarrow\{\forall \mathrm{X} \exists \mathrm{Y} \mathrm{R}(\mathrm{X}, \mathrm{Y}) \rightarrow \forall \mathrm{a} \\
& \exists \mathrm{b} \forall \mathrm{y}(\mathrm{Y} \epsilon \mathrm{b} \leftrightarrow \exists \mathrm{X}(\mathrm{X} \in \mathrm{a} \wedge \mathrm{R}(\mathrm{X}, \mathrm{Y}))) \rightarrow \\
& \forall \mathrm{X} \exists \mathrm{R}^{\gamma}(\beta)\left\{\left(\mathrm{X}^{\beta} \epsilon \mathrm{R}^{\gamma}(\beta) \wedge\left(\mathrm{P}(\beta) \subseteq \mathrm{X}^{\beta}\right)\right\}\right.
\end{aligned}
$$

Cet axiome combine l'axiome de remplacement et le principe de réflexion: l'ensemble des réflexions forme un ensemble noté $R(\omega)$ pour $\omega$ éléments.

Finalement, ces spéculations «métathéorétiques » n'ont pour but que de renouer avec celles de Cantor qui ont donné naissance à la théorie des ensembles. Mais de notre point de vue constructiviste, elles s'accompagnent d'un clin d'oeil sceptique.

Université de Montréal

* Notre modèle « del » n'a rien à voir avec les modèles booléens de Vopenka et Hajek (théorie des semi-ensembles). Nous avons défini récemment des «modèles fluctuants » pour notre théorie du continu, mais dans une perspective plus résolument constructiviste (à paraître dans un ouvrage qui doit être publié bientôt Fondements des Matbématiques. Introduction à une pbilosopbie constructiviste). 